\title{
TTR
}

Traduction, terminologie, rédaction

\section{Traduire au Québec la poésie étrangère : départager le rêve de la réalité}

\section{Mélanie Rivet}

Volume 28, numéro 1-2, 1er semestre-2e semestre 2015

La traduction littéraire et le Canada

Literary translation and Canada

URI : https://id.erudit.org/iderudit/1041656ar

DOI : https://doi.org/10.7202/1041656ar

Aller au sommaire du numéro

\section{Éditeur(s)}

Association canadienne de traductologie

ISSN

0835-8443 (imprimé)

1708-2188 (numérique)

Découvrir la revue

Citer cet article

Rivet, M. (2015). Traduire au Québec la poésie étrangère : départager le rêve de la réalité. TTR, 28(1-2), 181-205. https://doi.org/10.7202/1041656ar

\section{Résumé de l'article}

Le milieu de l'édition de poésie au Québec est intrinsèquement lié au contexte canadien et à son système de subventions publiques, à son bilinguisme français-anglais et à son bassin de lecteurs de poésie plutôt réduit. Dans ce contexte, les maisons d'édition sont susceptibles d'entretenir un rapport fragile avec la publication de poètes étrangers en traduction, et ce, même si cette activité a le potentiel d'enrichir le patrimoine culturel canadien. La présente recherche s'inscrit dans une réflexion pancanadienne sur la portée de la traduction littéraire dans l'éclosion, le maintien et la redéfinition de l'identité de la nation. Nous dressons ici un premier constat de la présence des poètes étrangers traduits en français et publiés au Québec entre janvier 2000 et octobre 2014. Nous avons cherché à répondre aux questions suivantes : 1) Quels poètes étrangers traduit-on? 2) Quelles sont les tendances qui se dessinent en matière de langue et de lieu d'origine? 3) Quelles maisons d'édition sont les plus engagées? 4) Qui traduit les poètes étrangers? 5) Quelles sont les sources de financement associées aux publications du corpus? Cette étude exploratoire pourra servir de base à d'autres études sur la traduction et l'édition de poésie étrangère au Québec. 


\title{
Traduire au Québec la poésie étrangère: départager le rêve de la réalité
}

\author{
Mélanie Rivet \\ Université du Québec en Outaouais
}

\begin{abstract}
Résumé
Le milieu de l'édition de poésie au Québec est intrinsèquement lié au contexte canadien et à son système de subventions publiques, à son bilinguisme français-anglais et à son bassin de lecteurs de poésie plutôt réduit. Dans ce contexte, les maisons d'édition sont susceptibles d'entretenir un rapport fragile avec la publication de poètes étrangers en traduction, et ce, même si cette activité a le potentiel d'enrichir le patrimoine culturel canadien. La présente recherche s'inscrit dans une réflexion pancanadienne sur la portée de la traduction littéraire dans l'éclosion, le maintien et la redéfinition de l'identité de la nation. Nous dressons ici un premier constat de la présence des poètes étrangers traduits en français et publiés au Québec entre janvier 2000 et octobre 2014. Nous avons cherché à répondre aux questions suivantes: 1) Quels poètes étrangers traduit-on? 2) Quelles sont les tendances qui se dessinent en matière de langue et de lieu d'origine? 3) Quelles maisons d'édition sont les plus engagées? 4) Qui traduit les poètes étrangers? 5) Quelles sont les sources de financement associées aux publications du corpus? Cette étude exploratoire pourra servir de base à d'autres études sur la traduction et l'édition de poésie étrangère au Québec.
\end{abstract}

Mots-clés: traduction littéraire, poésie étrangère, milieu de l'édition, Québec

Abstract

Poetry publishing in the province of Quebec is intrinsically linked to the Canadian context and its system of government subsidies, its FrenchEnglish bilingualism and its rather small pool of poetry readers. In this context, the publishers are likely to maintain a cautious relationship with the publication of foreign poets in translation, even if that activity has the potential to enrich Canada's cultural heritage. This research is part of a Canada-wide reflection on the role of literary translation in the birth, the preservation and the redefinition of a nation's identity. In this article, we share a preliminary observation of the presence of foreign poets translated into French and published in Quebec between January 2000 and October 2014. We sought to answer the following questions: 1) Which foreign poets are translated? 2) What trends are emerging in the field of language and place of origin? 3) What publishers are most involved? 4) Who translate foreign 
poets? 5) What are the funding sources behind the published corpus? This exploratory study could serve as a basis for further studies on translation and published foreign poetry in the province of Quebec.

Keywords: literary translation, foreign poetry, publishing industry, Quebec

\section{Introduction}

Le milieu de l'édition québécoise, intrinsèquement lié au système canadien de subventions publiques, au bilinguisme officiel du Canada et à son bassin de lecteurs de poésie plutôt réduit, peut pousser les maisons d'édition à entretenir un rapport fragile avec l'édition de poètes étrangers ${ }^{1}$ traduits. Lorsqu'elles font traduire de la poésie, c'est souvent sous l'égide d'un programme de subvention du gouvernement fédéral pour la traduction d'auteurs canadiensanglais en français ${ }^{2}$, car peu de soutien est accordé aux publications de poètes étrangers (Claxton, 2005). Pourtant, la publication d'œuvres de poètes étrangers traduites en français a le potentiel, par sa diversité, d'enrichir le patrimoine littéraire canadien. Comme l'expliquent Louis Jolicoeur (2010) et Gilles Pellerin (2010), la traduction littéraire joue en effet un rôle important au sein d'une littérature nationale en tant que moteur de diffusion et d'enrichissement d'une culture. Pour reprendre les mots de Louis Jolicoeur, «le rôle du traducteur est de faire connaître l'autre chez soi et de faire connaître les siens chez l'autre» (2010, p. 179).

Selon Mylène Dufault, «la traduction au Québec d'ouvrages littéraires d'auteurs issus de l'Amérique hispanique est encore un phénomène marginal. Pourtant, les entreprises de traduction de tels ouvrages se sont faites plus nombreuses au cours des vingt dernières

1. Nous entendons par «poètes étrangers» ceux et celles qui ne résident pas au Canada et qui n'y sont pas nés.

2. De tels programmes existent au Conseil des arts du Canada, comme le volet Traduction du programme «Rayonner à l'international», qui finance la traduction d'œuvres dramatiques et littéraires canadiennes en vue de les présenter ou de les publier à l'étranger, et le volet Traduction du programme «Rayonner au Canada», qui finance des activités liées à la traduction d'œuvres littéraires ou dramatiques en français, en anglais ou dans une langue autochtone. Dans ce dernier cas, les œuvres doivent être d'un auteur canadien et destinées à la publication ou à la diffusion au Canada. Ces programmes soutiennent la traduction et la diffusion de l'œuvre, non sa publication. Des programmes similaires existent au Québec, parmi lesquels le «Programme d'aide à l'exportation et au rayonnement culturel» de la Société de développement des entreprises culturelles (SODEC), qui vise l'achat de droits hors Québec de titres de littérature générale. 
années» (2013, p. i). Nous avons voulu explorer ce qu'il en était en matière de traduction de poésie. D'entrée de jeu, nous avons constaté qu'il existe peu de documentation récente sur le contexte dans lequel les maisons d'édition québécoises qui se consacrent à la poésie publient des traductions de poésie étrangère au Québec ${ }^{3}$. Nous espérons combler en partie cette lacune documentaire, en faisant écho aux publications qui s'intéressent à l'envers de la médaille, soit la diffusion de la littérature québécoise traduite et diffusée à l'étranger ${ }^{4}$.

Le présent article propose un portrait de la présence des poètes étrangers traduits en français et publiés au Québec entre janvier 2000 et octobre 20145. Ce portrait a été obtenu: 1) en dressant une liste exhaustive des poètes étrangers traduits; 2) en relevant les tendances observées en matière de langues et de cultures d'origine; 3) en identifiant les maisons d'édition québécoises les plus engagées; 4) en identifiant les traducteurs les plus actifs; 5) en explorant les moyens de financement dont ont bénéficié les ouvrages traduits, le cas échéant. Nous verrons que, malgré l'absence de soutien financier propre à la traduction et à la publication de poésie étrangère au Québec, il s'est publié nombre de titres au cours de la période étudiée, de divers auteurs étrangers, traduits par bon nombre de traducteurs, le tout, chez une poignée d'éditeurs québécois.

3. C'est le constat qui s'imposait à l'automne 2014, au moment où nous avons entrepris nos recherches. Nous avons pu repérer un mémoire de maîtrise intitulé La traduction de la littérature hispano-américaine au Québec: de l'intégration immigrante à la mondialisation éditoriale (Dufault, 2013), qui porte sur divers genres littéraires et sur certains auteurs de littérature hispano-américaine, particulièrement Yvonne América Truque (CÉDAH), Jaime Sabines (poésie, Écrits des Forges) et Horacio Castellanos Moya (Allusifs). Notons aussi la revue critique La Poésie au Québec produite par les Écrits des Forges qui, de 1989 à 1993, comportait une section consacrée à la poésie étrangère publiée au Québec (information repérée sur le site des Écrits des Forges).

4. Mentionnons sur ce thème l'article de Stratford et de Jolicoeur (2014).

5. Nous avons entrepris l'analyse de données en octobre 2014. Par ailleurs, nous avons fait commencer la période observée en janvier 2010 pour être en mesure de dresser un portrait de plus de dix ans de publications, ce qui nous semblait un minimum pour rendre compte des enjeux économiques et des ressources humaines affectées à ces publications dans les maisons d'édition. Bref, nous souhaitions dresser un portrait le plus juste et actuel possible au moment de la recherche et en témoigner de façon factuelle et descriptive. 


\section{Méthodologie}

Aux fins de notre étude, nous avons d'abord répertorié les maisons d'édition québécoises francophones actives qui se consacrent principalement à la poésie ou qui lui donnent une place prépondérante dans leur catalogue. Nous avons pour ce faire consulté les banques de données bibliographiques de Bibliothèque et archives nationales du Québec (BANQ) ainsi que les sites d'associations d'éditeurs (Association nationale des éditeurs de livres et Québec Édition) et de regroupements professionnels en poésie et en littérature (Maison de la poésie de Montréal, Union des écrivaines et des écrivains québécois, Association des traducteurs et traductrices littéraires du Canada). L'information recueillie dans ces sources a été validée sur les sites des maisons d'édition en question $^{6}$, dans des annonces parues dans les quotidiens La Presse et Le Devoir concernant des prix décernés à leurs auteurs ainsi que sur le site des diffuseurs de ces maisons (Prologue et Dimédia). $\mathrm{Au}$ terme de cette étape, nous disposions d'une liste des maisons d'édition québécoises francophones spécialisées dans la publication de poésie.

Nous avons ensuite consulté les catalogues de ces maisons d'édition afin de recueillir les données relatives à la publication de poètes étrangers traduits. Ces données ont été comparées à celles qui sont répertoriées dans l'Index Translationium de l'UNESCO, dans les bases de données de BANQ et de Bibliothèque et Archives Canada ainsi que sur le site Amazon.ca. Cela nous a permis d'assembler les données manquantes ou incomplètes.

Le profil sommaire des auteurs et des traducteurs a pour sa part été dressé à partir des fiches biographiques figurant sur les sites des maisons d'édition et dans le répertoire des membres de l'Association des traducteurs et traductrices littéraires du Canada.

Nous avons de surcroit distribué un questionnaire aux représentants des quatre maisons d'édition ayant publié des poètes étrangers en traduction française entre janvier 2000 et octobre 2014. Cette étape avait pour objectif de confirmer les données recueillies au préalable et d'obtenir des renseignements sur le mode de financement de la production des titres du corpus, le processus de sélection des traducteurs et des auteurs traduits, ainsi que sur les autres modes de soutien à la publication. Certains éditeurs nous

6. La maison Les Herbes rouges n'avait pas de site Internet en octobre 2014, au moment où la recherche a été effectuée. 
ont transmis leurs réponses par courriel. C'est le cas de Benoît Chaput, directeur général et littéraire de L'Oie de Cravan, de Paul Bélanger et Patrick Lafontaine, directeurs littéraires des Éditions du Noroît, ainsi que de Louise Trépanier, directrice administrative des Éditions d'art Le Sabord. Delphine Lefèvre, directrice administrative aux Écrits des Forges, a pour sa part répondu au même questionnaire lors d'une entrevue semi-dirigée réalisée au téléphone.

Les données relatives à la publication de poètes étrangers traduits, aux poètes en question et aux traducteurs ont ensuite été compilées à l'aide de tableaux synthèses, ce qui a permis de dégager des tendances en matière de pays d'origine des auteurs traduits, de langue source et de nombre de publications du corpus par éditeur, et de dresser un profil sommaire des traducteurs et des auteurs traduits. Cette série d'analyses a permis d'établir un constat de la publication de poètes étrangers en traduction française par les maisons d'édition québécoises.

Par ailleurs, nos recherches documentaires au sujet des auteurs visaient à dégager certains facteurs pouvant expliquer leur traduction. Sont-ils des auteurs contemporains ou du passé? Ont-ils reçu des prix? Quelle importance donne-t-on à leur œuvre dans leur pays ou au Québec? Pour les traducteurs, nous voulions dresser un portrait sommaire de leur profil, à commencer par savoir s'ils étaient aussi ou surtout auteurs, s'ils étaient membres de l'Association des traducteurs et traductrices littéraires du Canada et s'ils entretenaient un lien privilégié avec la maison d'édition (collaboration ponctuelle ou régulière), ayant en tête l'hypothèse que certains de ces facteurs pourraient influencer le choix des maisons d'édition de collaborer avec eux. Plusieurs autres éléments du profil des traducteurs auraient pu être relevés et pourraient faire l'objet d'une autre étude.

\section{Les maisons d'édition}

D'après nos recherches, vingt maisons d'édition ont publié de la poésie au Québec entre janvier 2000 et octobre 2014. De ce nombre, sept sont beaucoup plus actives que les autres et contribuent à bâtir le paysage québécois en matière de publication poétique. Il s'agit des maisons suivantes: les Écrits des Forges, les Herbes Rouges, l'Hexagone, les Éditions du Norôit, Poètes de brousse, Triptyque et le Lézard amoureux. Sept autres maisons d'édition publient aussi uniquement de la poésie, ou celle-ci fait bonne figure dans leur 
catalogue, bien que dans une moindre mesure: les Éditions d'art Le Sabord, Boréal, Cornac, les Éditions de l'Écrou, les Éditions du Passage, L'Oie de Cravan et La Peuplade. Finalement, nous avons relevé quelques œuvres poétiques dans le catalogue de six autres éditeurs québécois de plus petite taille ou qui donnent à la poésie un rôle secondaire dans leur démarche éditoriale: Christian Feuillette, De Courberon, la Grenouillère, Neige-galerie, Rodrigol et Ta mère ${ }^{7}$. Cette liste vise à dessiner un portrait actuel de l'édition de la poésie au Québec, selon les données auxquelles nous avons eu accès.

Nous concentrerons notre étude sur les publications des quatre maisons d'édition où sont parues, entre janvier 2000 et octobre 2014, l'ensemble des traductions recensées ${ }^{8}$ : les Écrits des Forges (Trois-Rivières; diffuseur Prologue), les Éditions du Noroît (Montréal; diffuseur Dimédia), L’Oie de Cravan (Montréal; diffuseur Dimédia) et les Éditions d'art Le Sabord (Trois-Rivières; diffuseur Dimédia). Nous avons recueilli les données suivantes au sujet des œuvres de notre corpus: titre des œuvres traduites et (si possible) originales; nom et profil des poètes traduits; nom et profil des traducteurs; pays d'origine et de résidence des auteurs et langues sources des œuvres. Cela nous a permis de dresser un portrait de la poésie étrangère traduite et publiée au Québec et de montrer, entre autres choses, la force de présence de certaines langues et de certains pays d'origine des auteurs traduits.

\section{Les poètes traduits et le nombre de publications par maison d'édition}

Nous avons compté 445 poètes étrangers traduits et publiés en français au Québec entre janvier 2000 et octobre 2014, dans 139 œuvres. Voici la répartition par maison d'édition ${ }^{9}$ :

7. En date du 30 août 2015, le site de cette maison d'édition indiquait qu'elle ne publiait plus de poésie.

8. Ont été exclues de l'étude les autopublications et les publications dans les magazines, puisque, d'une part, notre recherche s'inscrit dans la volonté de contribuer à produire des données chiffrées qui pourraient éventuellement mener à des prises de conscience des organismes soutenant le milieu de l'édition sur le contexte de la publication des oeuvres poétiques étrangères traduites. D'autre part, nous souhaitions circonscrire la recherche à un corpus défini et d'une taille raisonnable pour une analyse pertinente compte tenu du temps et des ressources dont nous disposions

9. Pour une liste complète des auteurs, voir l'Annexe 1. 
Écrits des Forges

Éditions du Noroît

L'Oie de Cravan

Éd. d'art Le Sabord
403 poètes, dont 109 publications individuelles, deux publications signées par deux coauteurs et 290 poètes regroupés en huit anthologies, pour un total de 119 œuvres, dont 88 en édition bilingue

36 poètes, dont 12 publications individuelles (trois du même poète), 25 poètes regroupés en deux anthologies (dont un aussi publié individuellement), pour un total de 14 œuvres

quatre poètes en publication individuelle, pour un total de quatre ouvres

deux poètes en publication individuelle, pour un total de deux œuvres

Nous observons que les Écrits des Forges est la maison qui a le plus investi dans la traduction de poésie étrangère depuis 2000, avec 119 publications ${ }^{10}$. Les Éditions du Noroît font aussi bonne figure avec 14 publications. Cette maison d'édition, bien qu'elle n'ait pas publié autant de poètes étrangers que les Écrits des Forges au cours de la période ciblée (403 poètes aux Écrits des Forges contre 36 pour le Noroît), est bel et bien engagée dans la publication de poésie étrangère, comme en témoigne sa collection Dialogues, qui "propose une rencontre entre des poètes du monde entier par le biais de la traduction ${ }^{11}$. L'Oie de Cravan et les Éditions d'art Le Sabord ne sont pas des joueurs importants, ayant respectivement publié seulement quatre et deux poètes étrangers en traduction

10. La maison les Écrits des Forges est aussi engagée dans la traduction vers le français d'auteurs québécois et canadiens non francophones pour diffusion au Québec, et elle contribue à la diffusion des poètes québécois en espagnol, principalement au Mexique (v. Stratford, 2008, n.p.; Stratford et Jolicoeur, 2014, p. 98-99). Dufault remarque que « [1]es échanges entre le Québec et le Mexique iront bon train pendant près de 20 ans. Si le principe de réciprocité est à la base de ces échanges et que chaque année on note un nombre similaire de parutions dans les deux directions, il demeure qu'au final, le nombre d'auteurs mexicains publiés au Québec entre 1992 et 2012 (65) est supérieur au nombre d'auteurs québécois publiés au Mexique pendant la même période (38)» (2013, p. 56).

11. Information repérée sur le site des Éditions du Noroît (http://www. lenoroit.com/site/index.php?section=3\&idCollection=6) le 20 octobre 2015. 
française au cours de la période étudiée. Nous les avons tout de même incluses dans notre étude, compte tenu de la petite taille de l'échantillon de maisons d'édition potentielles. Elles contribuent à valider ou à nuancer les données relatives aux Écrits des Forges et au Noroît.

Des auteurs étrangers traduits, nous notons très peu de récurrences dans les publications du corpus, sauf pour ceux qui suivent (entre parenthèses le nombre de livres du corpus dans lesquels ils sont inclus): Antoni Clapés (2); Tatiana Chtcherbina (2); Valerio Magrelli (2); Mercedès Roffé (3); Charles Simic (2).

\section{Pays d'origine ou de résidence des poètes traduits}

Aux Écrits des Forges, on compte 74 publications dont les auteurs résident au Mexique ${ }^{12}$, cinq dont les auteurs sont nés aux États-Unis ou y résident depuis un bon nombre d'années, quatre d'auteurs qui résident en Russie, quatre d'auteurs qui résident en Espagne, quatre d'auteurs qui résident en Argentine, en plus d'une ou deux publications d'auteurs provenant de l'un des 16 pays suivants: Australie, Allemagne, Belgique (œuvre originale en néerlandais), Chili, Chine, Colombie, Corée du Sud, Égypte, Grande-Bretagne, Grèce, Islande, Italie, Macédoine, Roumanie, Uruguay et Vénézuéla. Aux Éditions du Noroît, on trouve cinq publications dont l'auteur est né ou réside aux États-Unis depuis

12. La Foire du livre de Guadalajara choisissait en 2003 le Québec comme invité d'honneur, ce qui stimula les traductions entre le Mexique et le Québec. Au moins trois anthologies de poètes québécois traduits en espagnol furent publiées (ou copubliées) à cette occasion par les Écrits des Forges pour diffusion au Mexique (Stratford, 2008; Stratford et Jolicoeur, 2014). Dufault souligne l'importance que cette foire a eue dans les relations entre certains éditeurs du Mexique et les Écrits des Forges: «La présence régulière de Gaston Bellemare à la Foire du livre de Guadalajara fut [...] déterminante. Il y fit la connaissance des principaux acteurs du monde éditorial mexicain, principalement les institutions éditoriales académiques et étatiques (l'UNAM et le FCE) qui allaient devenir de fidèles partenaires de coédition » (2013, p. 55). Fait intéressant, notre corpus compte 17 traductions de poètes mexicains publiées avant 2003 aux Écrits des Forges, soit quatre en 2000, six en 2001, sept en 2002. La publication de poètes étrangers aux Écrits des Forges a donc commencé de façon régulière avant la Foire de Guadalajara. À compter de 2003, on remarque une légère hausse avec neuf traductions, puis six en 2004, huit en 2005, huit en 2006, sept en 2007, cinq en 2008. On observe ensuite une chute du nombre de poètes mexicains traduits: trois en 2009, quatre en 2010, deux en 2011, un en 2012, deux en 2013, deux en 2014. 
nombre d'années, trois auteurs qui résident au Mexique, deux auteurs qui résident en Espagne, deux en Italie, une publication dont l'auteur réside en Inde et une publication dont l'auteur réside en Corée. Aux éditions L'Oie de Cravan, on compte deux publications dont l'auteur est originaire des États-Unis, une dont l'auteur réside en Grèce et une dont l'auteur réside en République tchèque. Enfin, aux Éditions d'art Le Sabord, les deux publications répertoriées sont d'auteurs provenant des États-Unis. Notons que $55 \%$ des publications du corpus (77 sur 139) sont d'auteurs originaires du Mexique, $10 \%$ des États-Unis (14 sur 139), 4\% de l'Espagne (6 sur 139) et 31\% (49 sur 139) dont les provenances sont réparties entre l'Allemagne, l'Australie, la Belgique, le Brésil, le Chili, la Chine, la Colombie, la Corée, Cuba, la France, la GrandeBretagne, la Grèce, l'Islande, l'Italie, la Macédoine, la Roumanie, la Russie et l'Uruguay. En somme, il existe une diversité de pays d'origine des poètes traduits (25), et la provenance la plus marquée est le Mexique (et $96 \%$ des poètes mexicains traduits au Québec entre janvier 2000 et octobre 2014 l'ont été aux Écrits des Forges, réalité due aux partenariats et aux coéditions avec des éditeurs mexicains que les Écrits des Forges ont mis en place). Par ailleurs, chez l'ensemble des éditeurs du corpus, les États-Unis demeurent une source importante d'auteurs traduits; il s'agit même du lieu d'origine de l'entièreté des auteurs traduits aux Éditions d'art Le Sabord et de la moitié des auteurs (deux publications sur quatre) traduits chez L'Oie de Cravan.

\section{Les langues sources}

On compte pas moins de 14 langues sources traduites par les quatre éditeurs du corpus. Tous éditeurs confondus, les langues traduites en ordre décroissant selon le nombre de publications se déclinent comme suit: espagnol (99), anglais (14), catalan (4), russe (4), italien (4), coréen (3), allemand (2), grec (2), macédonien (2), roumain (2), chinois (1), islandais (1), néerlandais (1) et portugais (1). Il est à noter qu'une publication du Noroît est signée par un poète originaire de l'Inde, mais que ce dernier a écrit l'œuvre originale en langue anglaise. La même chose se produit chez L'Oie de Cravan, où un auteur qui réside en République tchèque a écrit en langue anglaise. Malgré la diversité de langues sources (14) dans le corpus, l'espagnol se démarque nettement comme la langue la plus traduite, provenant principalement du Mexique, et l'anglais comme deuxième langue dominante, provenant surtout des 
États-Unis, mais aussi, de façon moins importante, de l'Australie (une anthologie), de l'Inde (une publication) et de la République tchèque (une publication).

\section{Tendances : profils et choix}

Les profils des poètes traduits par les maisons d'édition sont divers. Ce sont parfois des auteurs contemporains, parfois des icônes du passé. Par exemple, les Écrits des Forges privilégient les poètes actuels. En effet, cette maison collabore avec des maisons d'édition mexicaines et des organismes du Mexique afin de dynamiser les échanges entre les littératures mexicaines et québécoises actuelles, entre autres par le Festival de poésie de Trois-Rivières et le prix Gatien-Lapointe-Jaime-Sabines. Ce prix s'adresse aux auteurs actuels qui ont à leur nom au moins un ouvrage de poésie traduit dans le pays partenaire. Ce prix suscite la publication d'auteurs mexicains par les Écrits des Forges (souvent en coédition avec une maison mexicaine en édition bilingue). Ces activités n'empêchent pas les Écrits des Forges de publier certaines œuvres de poètes marquants du passé ${ }^{13}$, comme le montre notre corpus avec la présence, entre autres, de Jaime Sabines et Pablo Neruda. Les Écrits des Forges publient aussi des anthologies de poètes actuels peu diffusés hors de leur propre culture (provenant de l'Islande et de la Chine, par exemple).

Une caractéristique commune semble se dégager chez l'ensemble des auteurs traduits: ils ont été jugés importants au sein de leur propre littérature nationale, soit parce qu'ils ont écrit une longue liste d'ouvrages, soit parce qu'ils ont reçu une forme de reconnaissance (prix), soit parce qu'ils jouissent déjà d'une renommée internationale (Edgar Allan Poe, par exemple). Aux Éditions d'art Le Sabord, les deux poètes traduits, Ilya Kaminsky et Mariela Griffor, entretiennent un lien privilégié avec leur traducteur, Guy Jean. En effet, Mariela Griffor a publié, au sein de sa propre maison d'édition aux États-Unis (Marick Press), une œuvre originale de Guy Jean, dans une traduction d'Ilya Kaminsky. Lorsqu'interrogés sur la façon dont se faisait le choix des auteurs

13. «La première (Écrits des Forges) a publié en français de nombreux canons de la poésie mexicaine [...]. Les efforts de ces éditeurs ont été précédés d'un premier mouvement d'accueil de la littérature hispanoaméricaine, mouvement principalement lié à la venue, au Canada, d'auteurs hispano-américains immigrants, souvent des réfugiés qui avaient fui la guerre ou la dictature dans leur pays d'origine.» (Dufault, 2013, p. i) 
étrangers à publier, les représentants des Écrits des Forges ont affirmé que les propositions venaient soit d'une recommandation de leur réseau concernant une œuvre de grande qualité, ou encore de recommandations d'une maison d'édition étrangère, partenaire de diffusion. Les représentants des Éditions du Noroît ont affirmé que la plupart des publications du corpus étaient nées d'une démarche initiée par un traducteur. Aux Editions d'art Le Sabord, la publication des deux œuvres a été influencée par une amitié littéraire entre le traducteur et les auteurs, et entre l'éditeur de l'époque et le traducteur. Dans le cas de L'Oie de Cravan, son directeur littéraire nous confiait que les projets s'étaient d'abord présentés

au fil des amitiés. Dans le cas des poèmes de Poe, il s'agit d'une réédition d'un livre d'abord paru en 1997 aux éditions Le temps qu'il fait. Alice Becker-Ho m'a proposé de le rééditer, ce que j'ai accepté avec plaisir. En ce qui concerne Katerina Iliopoùlou, un poète ami (Jean-Yves Bériou) m'avait parlé de cette poésie, pratiquement inédite en français. Il m'a mis en contact avec le traducteur Michel Volkovitch qui a eu la gentillesse de traduire le livre. [...] Je connaissais depuis longtemps la traduction de Alice Becker-Ho et je l'appréciais. Michel Volkovitch est sans doute le principal traducteur de poésie du grec à la langue française en ce moment, ce fut un honneur de travailler avec lui. (Chaput, mars 2015) ${ }^{14}$

\section{Les traducteurs}

Les traducteurs d'œuvres littéraires étrangères sont nombreux au Québec. Nous avons relevé 70 traducteurs dans le corpus: 42 actifs aux Écrits des Forges entre 2000 et 2014, dont 17 ont travaillé en équipe; 23 aux Éditions du Noroît, dont quatre ont travaillé en équipe et 16 ont participé à une anthologie; quatre aux éditions de L'Oie de Cravan et un seul aux Éditions d'art Le Sabord ${ }^{15}$. De ce nombre, les plus actifs sont aux Écrits des Forges: Françoise Roy (avec 38 traductions), le duo Émile et Nicole Martel (compilant 20 traductions individuelles ou en équipe); Dominique Soucy (sept traductions), François-Michel Durazzo (six traductions),

14. En réponse aux questions 4 et 5 du questionnaire aux éditeurs : Comment s'est fait le choix des auteurs traduits et publiés? Et comment s'est fait le choix des traducteurs?

15. Pour une liste complète des traducteurs, voir l'Annexe 2. 
Denys Bélanger (quatre traductions). Le nombre de collaborations permet de dégager un lien de fidélité entre ces traducteurs et les Écrits des Forges. Les Éditions du Noroît entretiennent aussi un lien de fidélité avec certains traducteurs du corpus: Nelly Roffé et Daniel Canty (trois traductions chacun), François-Michel Durazzo et Francis Catalano (deux traductions chacun). La taille réduite du corpus de cette maison pourrait nous faire douter du caractère éloquent de ces chiffres; cependant, le représentant de la maison nous a confié que les directeurs littéraires travaillaient en étroite collaboration avec des gens de «leur famille littéraire», des traducteurs en qui ils ont confiance et avec lesquels un lien est déjà établi. Le corpus des éditions L'Oie de Cravan et des Éditions d'art Le Sabord ne permet pas l'analyse de la présence d'un lien de fidélité entre les traducteurs et l'éditeur. Nous avons, en outre, relevé seulement trois traducteurs qui ont travaillé dans plus d'une des maisons d'édition du corpus. En effet, Francis Catalano, FrançoisMichel Durazzo et Nelly Roffé ont tous trois traduit pour les Écrits des Forges et le Noroît. Le passage d'une maison d'édition à l'autre semble donc plutôt rare, du moins, en ce qui concerne les éditeurs du corpus, ce qui soutient le concept de famille littéraire énoncé par le représentant des Éditions du Noroît.

Nous avons noté qu'au moins huit des 23 traducteurs des Éditions du Noroît sont aussi, sinon surtout, des poètes maintes fois publiés. Parmi ceux-ci, mentionnons Normand Baillargeon (essai, poésie et ouvrages critiques), Daniel Canty (dramaturgie, fiction télévisée), Francis Catalano (poésie), Denise Desautels (poésie, livres d'artiste et récit), Antonio d'Alfonso (poésie), FrançoisMichel Durazzo (poésie et essai), Pierre Nepveu (poésie et essai) et Louis Jolicoeur (roman, récit, essai et nouvelles). Plusieurs des traducteurs actifs aux Écrits des Forges sont eux aussi poètes ou écrivains. Mentionnons, entre autres, Christine Balta (poésie), Pierre Des Ruisseaux (poésie), André Doms (poésie et essai), François-Michel Durazzo (poésie et essai), Émile Martel (poésie et conte), Nicole Martel (roman), Jean Portante (poésie), Françoise Roy (roman et poésie). Le seul traducteur associé aux Éditions d'art Le Sabord, Guy Jean, est un poète d'expérience qui traduit peu. Notons aussi que des 70 traducteurs du corpus, seulement quatre étaient membres de l'Association des traducteurs et traductrices littéraires du Canada (ATTLC) lors de la collecte initiale de 
données en octobre 2015 ${ }^{16}$ : Francis Catalano, Antonio d'Alfonso, Charlotte Melançon et Nelly Roffé. En date du 27 mai 2017, ces quatre traducteurs n'étaient plus membres de l'ATTLC. De notre corpus, seul Louis Jolicoeur figurait au répertoire de l'ATTLC à cette date ${ }^{17}$. Un portrait plus détaillé des traducteurs littéraires pourrait faire l'objet d'une étude complémentaire à celle-ci.

\section{Sources de financement}

Les quatre éditeurs du corpus ont affirmé avoir principalement financé leurs publications de poètes étrangers traduits en français à même leur fonds de roulement. Ils y ont injecté leurs ressources sans soutien spécifique de l'État, hormis à quelques occasions (non quantifiées par les représentants des maisons d'édition), soit par une petite subvention (aucun détail donné) ou une réaffectation d'une partie des subventions reçues pour le fonctionnement ou d'autres projets, qu'ils ont pu réattribuer à la publication d'une traduction. Ce geste demeure rare selon les éditeurs du corpus. Les Éditions du Noroît bénéficient aussi du Club des amis du Norôit ${ }^{18}$, qui les «aide en partie à faire les frais d'un livre par année ${ }^{19}$. La publication des œuvres du corpus tient donc surtout, selon les Écrits des Forges, les Éditions du Noroît et de L'Oie de Cravan, d'un engagement dans leur rôle d'éditeur et d'acteur de la littérature. Les copublications réalisées par les Écrits des Forges et les Éditions du Noroît avec des maisons d'édition du Mexique ou de la France sont en fait des codiffusions d'auteurs issus de leur littérature respective. Ces partenariats ne contribuent pas financièrement à

16. Selon la consultation du répertoire des membres en ligne en date du 20 octobre 2015. Repéré au http://attlc-ltac.org/fr/directory_members.

17. La recherche a été effectuée par nom et par le filtre de recherche du genre littéraire poésie, dans lequel figurent pourtant 24 membres sur les 126 que compte le répertoire de l'Association.

18. «Le Club des ami. e. s consiste en un fonds financier constitué grâce aux dons du public qui permet au Noroît d'élargir ses activités éditoriales. Cet engagement généreux envers la poésie construit une solidarité parallèle et précieuse qui contribue à l'innovation et à la réalisation de nombreux projets, tels que le Bulletin du Noroît, la publication de livres développés conjointement avec des artistes, la traduction d'auteurs étrangers et la tenue de rencontres et de spectacles littéraires.» (Repéré au http://test.lenoroit. com/wordpress/club-des-amis/ le 27 mai 2017)

19. Réponse du directeur littéraire et du directeur général des Éditions du Noroît au questionnaire aux éditeurs (Bélanger et Lafontaine, mars 2015). 
la publication de poètes étrangers ${ }^{20}$. Des quatre éditeurs du corpus, trois ont clairement émis le souhait qu'un soutien financier soit mis en place pour appuyer ce type de parutions et que les options de financement, si elles existent, soient mieux diffusées auprès d'eux ${ }^{21}$. Delphine Lefèvre, directrice administrative aux Écrits des Forges, commente notamment:

Le gouvernement devrait augmenter ce soutien, car il est enrichissement de notre littérature. Les échanges avec le Mexique ont créé une certaine "poésie de l'échange» entre le Québec et le Mexique. Ces régions du monde ont en commun énormément en matière de poésie, et en facteurs de vie, et ces échanges ont fait croitre cette poésie. Plusieurs sujets traités sont communs, comme les langues, les frontières, les indigènes... Sans l'action de notre maison d'édition, ces liens n'auraient pas existé, et ces deux littératures n'auraient pas été autant stimulées. Ces échanges ne peuvent que faire accroitre la qualité de la littérature des deux côtés, et celle du monde. (Lefèvre, mars 2015)

Louise Trépanier, responsable administrative, distribution et diffusion aux Éditions d'art Le Sabord, a pour sa part affirmé qu'aucune autre publication de traduction étrangère ne serait envisagée par son équipe éditoriale tant qu'un financement approprié ne serait pas offert, car les ventes de tels livres ne couvrent pas les investissements engagés:

Les ventes de poésies sont déjà difficiles avec nos propres auteurs québécois, les ventes de poètes inconnus ici encore plus. Il n'existe aucun soutien financier actuellement, ce qui nous empêche de continuer à réaliser de tels projets, car la survie de la maison d'édition serait menacée. Les seuls poètes étrangers qui pourraient être publiés (pour couvrir leurs frais) seraient les très grands poètes reconnus, et encore. (Trépanier, mars 2015)

20. Conclusion tirée de l'entretien avec la représentante des Écrits des Forges (Lefèvre, mars 2015) et des réponses fournies par les représentants des Éditions du Noroît (Bélanger et Lafontaine, mars 2015).

21. «[À] ma connaissance, il y a très peu d'aide de disponible au Québec et au Canada (je serais content d'être informé si vous connaissez quelque chose!). On me dit qu'on peut avoir des subventions de certains des pays d'origine des poètes.» Réponse fournie par le directeur général et littéraire de L'Oie de Cravan, Benoît Chaput (mars 2015). 
Toute l'activité éditoriale que nous avons recensée ici semble donc être le fruit de l'engagement des éditeurs dans l'enrichissement de la littérature de leur milieu, quelques fois motivée par des amitiés littéraires.

\section{Conclusion}

Le portrait de la poésie étrangère traduite et publiée au Québec entre janvier 2000 et octobre 2014 se présente donc ainsi en quelques chiffres: 139 œuvres publiées, 445 poètes étrangers, 25 pays d'origine ou de résidence et 14 langues sources. Les publications qui permettent la diffusion des poètes étrangers se présentent comme suit: $7 \%$ sont des anthologies; $71 \%$ sont des traductions de l'espagnol et $10 \%$ de l'anglais; $55 \%$ sont d'auteurs qui résident au Mexique, $10 \%$ sont d'auteurs qui résident aux Etats-Unis. Sur les 70 traducteurs du corpus, dix ont plus d'un titre publié. Par ailleurs, 119 des 139 œuvres du corpus (86\%) ont été publiées par les Écrits des Forges, et 14 (10\%) l'ont été par les Éditions du Noroît. Il semble qu'aucune subvention spécifique ne soit allouée à ces projets.

Il existe donc une pratique réelle de publication de poésie étrangère au Québec qui donne à lire une certaine diversité d'auteurs étrangers, actuels ou du passé. Cette pratique a plusieurs teintes et elle couvre tous les continents. Elle est rendue possible grâce à l'engagement financier et artistique d'un petit groupe déditeurs actifs et de traducteurs engagés, souvent liés soit aux auteurs traduits ou à la culture/langue d'origine. Nos observations permettent aussi de souligner la situation particulière des Écrits des Forges dans le paysage québécois ainsi que leur rapport étroit avec le Mexique et le lien privilégié qu'ils ont tissé avec les traducteurs littéraires qu'ils publient. Les Écrits des Forges ont une pratique de coédition avec le Mexique, qui est pour les Écrits des Forges un partenaire de diffusion biculturelle très actif ${ }^{22}$. Il existe un certain vide quant au financement de ce type de publications au Québec actuellement ${ }^{23}$, vide que les éditeurs des Écrits des Forges, des Éditions d'art Le Sabord et de L'Oie de Cravan souhaitent voir

$\overline{22 .}$ Les Écrits des Forges ont confirmé leurs liens avec le Mexique en 2005 dans le cadre du Festival de poésie de Trois-Rivières (v. Stratford, 2008).

23. Des coéditions bilingues peuvent recevoir un financement de deux États: celui du poète québécois, et celui du poète mexicain par exemple. C'est un mode de fonctionnement qui soutiendrait, du moins en partie, le coût des publications (Dufault, 2013, p. 96). 
comblé $^{24}$. Cette étude préliminaire sème des pistes pour d'autres études qui soutiendront la création d'un portrait détaillé de la traduction d'œuvres poétiques étrangères au Québec depuis 2000. Nous souhaitons que le constat du manque de documentation sur le contexte de la publication de poésie étrangère au Québec, ainsi que celui du manque de soutien financier offert à ce type de publications permettent à d'autres chercheurs de s'appuyer sur des chiffres pour explorer plus à fond ce terrain de recherche et éventuellement soutenir une prise de conscience collective de l'importance de ce type de publication et des conditions dans lesquelles elles sont réalisées. Ultimement, ces recherches pourront contribuer à stimuler l'évolution positive des conditions de la publication de poètes étrangers traduits en français au Québec.

\section{Références}

Bélanger, Paul et Patrick Lafontaine (mars 2015). Réponses au questionnaire "Publication de traductions de poésie étrangère au Québec entre 2000 et 2014 » préparé par Mélanie Rivet.

Chaput, Benoît (mars 2015). Réponses au questionnaire «Publication de traductions de poésie étrangère au Québec entre 2000 et 2014» préparé par Mélanie Rivet.

Claxton, Patricia (2005). "La traduction littéraire au Canada». Communication présentée au Congrès de l'OTTIAQ, 25 novembre 2005, dans le cadre de la table ronde L'Édition, milieu aux multiples horizons. [http://www.attlc-ltac.org/fr/la-traduction-litteraire-aucanada] (consulté le $1^{\text {er }}$ octobre 2014).

Dufault, Mylène (2013). La traduction de la littérature hispano-américaine au Québec: de l'intégration immigrante à la mondialisation éditoriale. Mémoire de maîtrise, Université de Montréal. [http://hdl.handle. net/1866/9830] (consulté le 20 octobre 2015).

Jolicoeur, Louis (2010). «Traduction littéraire et diffusion culturelle: entre esthétique et politique». Cabiers franco-canadiens de l'Ouest, 22, 2, p. 177-196.

Lefèvre, Delphine (mars 2015). Réponses au questionnaire «Publication de traductions de poésie étrangère au Québec entre 2000 et 2014» préparé par Mélanie Rivet.

Nadeau, Marc-André (1988). Lévaluation de programme: théorie et pratique. Québec, Les Presses de l'Université Laval.

Pellerin, Gilles (2010). «Influence argentine sur la nouvelle québécoise». Nuit blanche, 120, p. 67-73.

24. Selon les entrevues réalisées avec les éditeurs en mars 2015. 
Poupaud, Sandra, Anthony Pym et Ester Torres Simón (2009). «Finding Translations. On the Use of Bibliographical Databases in Translation History». Meta, 54, 2, p. 264-278.

Stratford, Madeleine (2008). «Moi, mes souliers ont beaucoup voyagé... en Amérique du Nord: diffusion de la poésie québécoise de langue française traduite en espagnol». Cycle de conférences midis du Département de linguistique et de traduction. Université de Montréal, mars.

Stratford, Madeleine et Louis Jolicoeur (2014). «La littérature québécoise traduite au Mexique: trois anthologies à la Foire du livre de Guadalajara de $2003 »$. Meta, 59, 1, p. 97-123.

Trépanier, Louise (2015). Réponses au questionnaire «Publication de traductions de poésie étrangère au Québec entre 2000 et 2014» préparé par Mélanie Rivet.

\section{Sites des maisons d'édition du corpus}

Éditions d'art Le Sabord. [http://www.lesabord.qc.ca/] (consulté le $1^{\text {er }}$ octobre 2014).

Éditions du Noroît. [http://www.lenoroit.com/] (consulté le 1 $1^{\text {er }}$ octobre 2014).

Les Écrits des Forges. [http://www.ecritsdesforges.com/] (consulté le $1^{\text {er }}$ octobre 2014).

L'Oie de Cravan. [http://www.oiedecravan.com/] (consulté le $1^{\text {er }}$ octobre 2014).

\section{Sites des autres maisons d'édition québécoises publiant de la poésie (ou de leurs diffuseurs)}

Christian Feuillette. [http://www.feuillette.ca/] (consulté le 1er octobre 2014).

Diffusion Dimédia. [http://www.dimedia.com/] (consulté le 1er octobre 2014).

Éditions Cornac. [http://editionscornac.com/] (consulté le $1^{\text {er }}$ octobre 2014).

Éditions De Courberon. [http://www.decourberon.com/] (consulté le $1^{\text {er }}$ octobre 2014).

Éditions de La Grenouillère. [http://www.delagrenouillere.com/] (consulté le $1^{\text {er }}$ octobre 2014).

Éditions de l'Écrou. [http://lecrou.com/] (consulté le 1 $1^{\text {er }}$ octobre 2014).

La Peuplade. [http://lapeuplade.com/] (consulté le $1^{\text {er }}$ octobre 2014).

Le lézard amoureux. [http://www.livresquebecois.com/editeurs. asp?id=308] (consulté le $1^{\text {er }}$ octobre 2014). 
Les Éditions de l'Hexagone (Québécor Media).[http://www.edhexagone. $\mathrm{com} /]$ (consulté le $1^{\text {er }}$ octobre 2014).

Les Éditions de Ta Mère. [http://www.tamere.org/] (consulté le $1^{\text {er }}$ octobre 2014).

Les Éditions Rodrigol. [http://leseditionsrodrigol.com/html/CMS/] (consulté le $1^{\text {er }}$ octobre 2014).

Les Éditions Triptyque. [http://www.triptyque.qc.ca] (consulté le $1^{\text {er }}$ octobre 2014).

Poètes de brousse. [http://www.poetesdebrousse.org] (consulté le $1^{\text {er }}$ octobre 2014).

\section{Organismes professionnels et associations}

Association des traducteurs et traductrices littéraires du canada. [http:// attlc-ltac.org/] (consulté le $1^{\text {er }}$ octobre 2014).

Association nationale des éditeurs de livres. [http://www.anel.qc.ca/] (consulté le $1^{\text {er }}$ octobre 2014).

Maison de la poésie (Montréal). [http://www.maisondelapoesie.qc.ca/] (consulté le $1^{\text {er }}$ octobre 2014).

Québec Édition. [http://quebecedition.qc.ca/] (consulté le $1^{\text {er }}$ octobre 2014).

Union des écrivains du Québec. [http://uneq.ca/] [(consulté le $1^{\text {er }}$ octobre 2014).

\section{Sources documentaires et bases de données bibliographiques}

Bibliothèques et Archives Canada. [http://www.bac-lac.gc.ca/fra/Pages/ accueil.aspx\#tab-5] (consulté le $1^{\text {er }}$ octobre 2014).

Bibliothèques et Archives nationales du Québec. [http://www.banq. qc.ca/] (consulté le $1^{\text {er }}$ octobre 2014).

Festival de poésie de Trois-Rivières. [http://www.fiptr.com/] (consulté le $1^{\text {er }}$ octobre 2014).

Index Translatonium de l'UNESCO. [http://www.unesco.org/xtrans/] (consulté le $1^{\text {er }}$ octobre 2014).

Mélanie Rivet 646-B, boul. des Hautes-Plaines Gatineau (Québec) J8Z 2W1 CANADA artatoi@gmail.com rivm17@uqo.ca 


\section{Annexe 1 : Liste des poètes étrangers traduits et publiés au Québec}

Les Écrits des Forges : publications individuelles ou par des coauteurs

\begin{tabular}{|c|c|c|c|}
\hline \multirow[t]{8}{*}{2000} & Óscar Oliva & 2002 & Saúl Ibargoyen \\
\hline & María Baranda & & Kata Kulavkova \\
\hline & Justo Jorge Padron & & Víctor Manuel Mendiola \\
\hline & Jorge Souza & & Francisco Magaña \\
\hline & Víctor Sandoval & & Luan Starova \\
\hline & Salima Ait Mohamed & & Juan Gelman \\
\hline & Irina Petraş & & Patricia Medina \\
\hline & & & Tatiana Oroño \\
\hline \multirow[t]{26}{*}{2001} & Antonio Deltoro & & Jerome Rothenberg \\
\hline & Ana María Jamarillo & & Bernardo Ruiz \\
\hline & Daniel Samoilovich & & Luis Armenta Malpica \\
\hline & Alicia Genovese & & \\
\hline & Juan José Macias & 2003 & -- \\
\hline & Reynaldo Valinho Alvarez & & \\
\hline & $\begin{array}{l}\text { Armando Alanís Pulido } \\
\text { Fabio Morábito }\end{array}$ & 2004 & Myriam Montoya \\
\hline & Gabriel Zaïd & 2005 & Gabriela Botti \\
\hline & Coral Bracho & & Tatiana Chtcherbina \\
\hline & Francisco Hernandez & & Pablo Neruda \\
\hline & Tchékov & & Francesca Parcerisas \\
\hline & Óscar Oliva & & Eduardo Hurtado \\
\hline & María Baranda & & Valerio Magrelli \\
\hline & Justo Jorge Padron & & Juan José Macías \\
\hline & Jorge Souza & & Margarita Mendoza Palomar \\
\hline & Víctor Sandoval & & Guillermo Fernández \\
\hline & Salima Ait Mohamed & & Luis Aguilar \\
\hline & Irina Petraş & & Calin Vlasie \\
\hline & Juan José Macias & & Jaime Augusto Shelley \\
\hline & Reynaldo Valinho Alvarez & & Sergio Briceño González \\
\hline & Armando Alanís Pulido & & Carles Duarte \\
\hline & Fabio Morábito & & Patricia Velasco \\
\hline & Gabriel Zaid & & Eli Tolaretxipi \\
\hline & Coral Bracho & & Jorge Humberto Chavez \\
\hline & Francisco Hernandez & & Raul Banuelos Salcedo \\
\hline & Tchékov & & \\
\hline
\end{tabular}


2005 Luis Armenta Malpica

Antonio Marts

Rafael Cadenas

Marco Antonio Campos

Vicente Quirarte

Lucero Alanis de Gurrola

León Plascencia Nol

Jo Ji-hum

Gabriel Martín

Ken Norris

Jaime Sabines

Elsa Cross

Miguel García Ascensio

Luis Fernando Ortega

Francisco Segovia

Cho Byunghwa

\section{$2006--$}

2007 Pierre Joris

Juan Carlos Galeano

Maria Baranda

Hugo Plascencia Madrid

Rosa Alice Branco

Lucero Alanis de Gurrola

Armando Alanís Pulido

Luis Armenta Malpica/

Ricardo Quijano

Jorge Esquinca

Boris Pasternak

Erich Wolfgang Skwara

Elva Macías

Diana Lichy

Sylvia Vainberg

Luis Alberto Arellano

Arturo Ipiéns

Paolo Ruffilli

Enriqueta Ochoa

Julio Trujillo

Blas Roldán

Patricia Medina

Andrea Montiel

Carlos Vicente Castro
2007 Tatiana Chtcherbina

Pablo Neruda

Francesc Parcrisas

Eduardo Hurtado

Valerio Magrelli

Juan José Macías

Jaime Sabines

Elsa Cross

Miguel García Ascensio

Luis Fernando Ortega

Francisco Segovia

Cho Byunghwa

2008 Minerva M. Villarreal

Enrique Cortazar

Antoni Clapés

Luis Vicente de Aguinaga

José Javier Villarreal

Ricardo Castillon

2009 Baudelio Camarillo

César Silva Márquez

Luis Dominguez

C. Schvartz/G. Manfredi

2010 José Àngel Leyva

Ernesto Lumbreras

José Landa

Abigael Bohorquez

2011 Miguel Àngel Andrade

Miguel Maldonado

2012 Ricardo Solís

Stefaan Van Den Bremt

Rodolfo Häsler

2013 Juan Castañed

Rodriguez Diez César

2014 Gaëlle Le Calvez

Mónica Mansour 


\section{Les Écrits des Forges: anthologies}

200341 poètes de la Grande-Bretagne

Jorge Cuesta rassemble 22 poètes, alors âgés de 24 à 75 ans dans le Mexique de la fin des années 1920

2004 Shi Bo a regroupé et traduit 120 femmes poètes, de la Chine ancienne à la Chine d'aujourd'hui Thór Stefánsson a regroupé 25 poètes islandais

2005 Evgueni Bounimovitch rassemble 33 poètes russes contemporains en deux vagues (dont K. Kedrov, I. Jdanov, A. Dragomochtchenko, L. Rubinstein, T. Chtcherbina et Bounimovitch, et D. Bykov, I. Gougolev, M. Ameline, P. Barskova, D. Davydov et V. Pavlova)

Anthologie de 48 poètes catalans qui publient depuis les trente dernières années

2006 Philip Hammial a regroupé les textes de 25 poètes australiens: Adam Aitken, John Anderson, Bruce Beaver, Michael Brennan, David Brooke, Pam Brown, Joanne Burns, MTC Cronin, Richard Deutch, Brook Emery, John Forbes, Carolyn Gerrish, Philip Hammial, Martin Langford, Kerry Leves, Emma Lew, Kate Liley, Jennifer Maiden, Leith Morton, Lizz Murphy, Zan Ross, Jutta Sieverding, John Tranter, Deborah Westbury, Les Wicks

200817 poètes allemands contemporains, nés entre 1929 et 1982: Andreas Altmann; Nora Bossong; Hans Georg Bulla; Tanja Dückers; Walter Helmut Fritz; Peter Härtling; Gisela Kraft; Björn Kuhligk; Axel Kutsch; Ulrike Almut Sandig; Hans Dieter Schäfer; Silke Andrea Schuemmer; Ludwig Steinherr; Sabine Techel; Sandra Trojan; Ron Winkler; Annemarie Zornack 
Les Éditions du Noroît: publications individuelles

$\begin{array}{llrl}2000 & \text { Valerio Magrelli } & 2009 & \begin{array}{l}\text { Mercedes Roffé } \\ 2001\end{array} \text {-- } \\ 2002 & -- & & \text { Jose Luis Rivas } \\ 2003 & - \text { Antonio Porta } \\ 2004 & \text { Mercedes Roffé } & 2010 & \text { Charles Simic } \\ 2005 & -- & 2011 & \text { Carmen Yáñez } \\ 2006 & \text { Sivia Pratt } & 2012 & -- \\ 2007 & -- & 2013 & \text { Mercedes Roffé } \\ 2008 & \text { Rabindranath Tagore } & 2014 & \text { Antoni Clapés }\end{array}$

\section{Les Éditions du Noroît : anthologies}

2005 Han Daekyun regroupe 12 poètes coréens contemporains

2013 Antoine Boisclair regroupe 13 poètes américains contemporains: John Ashbery; Amy Clampitt; Robert Creeley; Louise Glück; Fanny Howe; William Merwin; John Montague; Eric Ormsby; Michael Palmer; Charles Simic; Mark Strand; Richard Wilbur; Charles Wright

\section{Les Éditions L'Oie de Cravan: publications individuelles}

2009 Shane Brangan

2011 Michael Hurley

2012 Katerina Iliopoùlou

2013 Edgar Allan Poe

\section{Les Éditions d'art Le Sabord : publications individuelles}

2010 Ilya Kaminsky

2012 Mariela Griffor 


\section{Annexe 2: Liste des traducteurs ayant signé les traductions du corpus $^{25}$}

\section{Les Écrits des Forges}

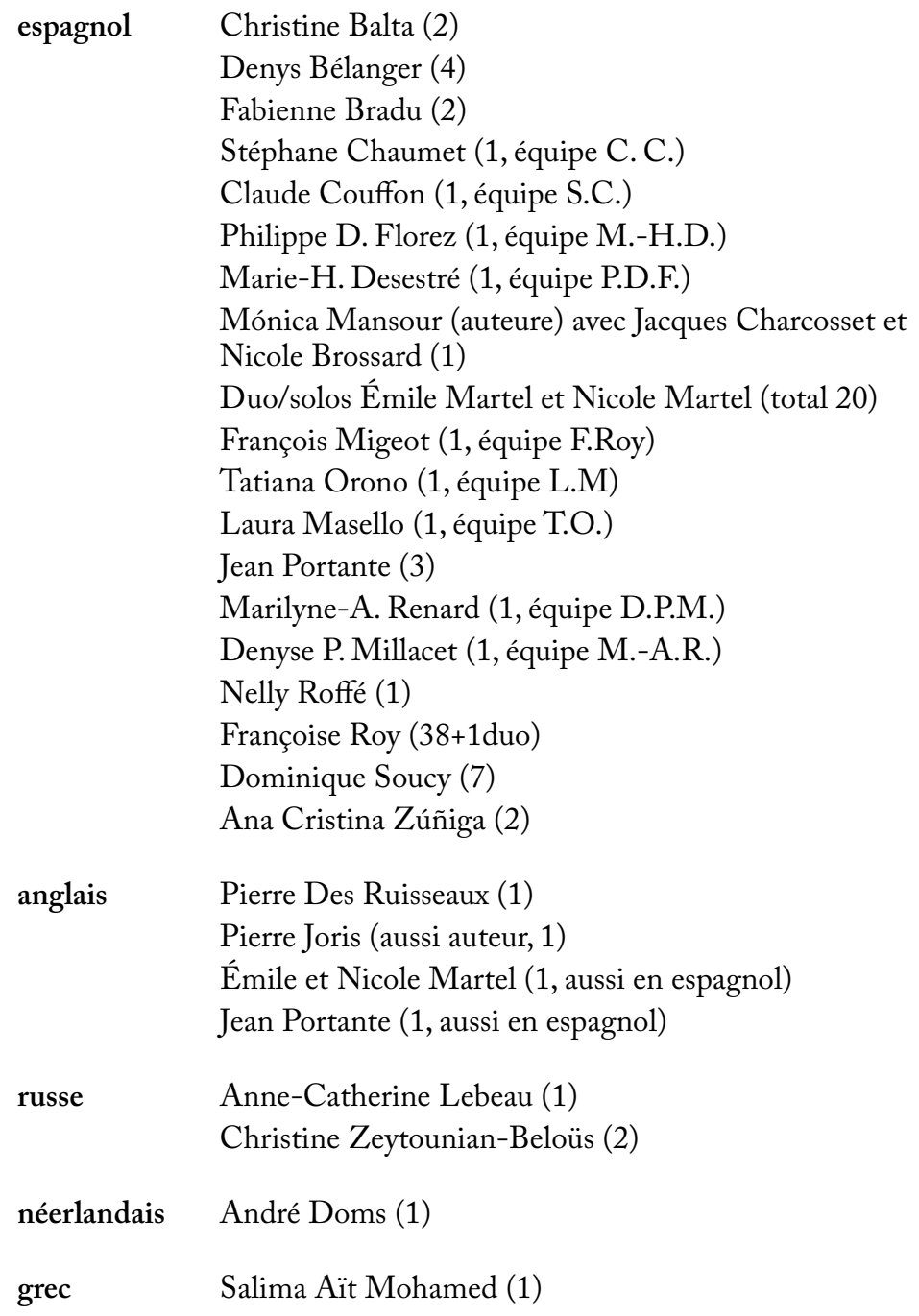

25. Figure entre parenthèses le nombre d'œuvres traduites. Les traducteurs dont le nom est souligné sont ceux qui figuraient dans la liste des membres actifs de l'ATTLC au 20 octobre 2015. Aucun ne figurait dans la liste des membres actifs du répertoire au 27 mai 2017. 


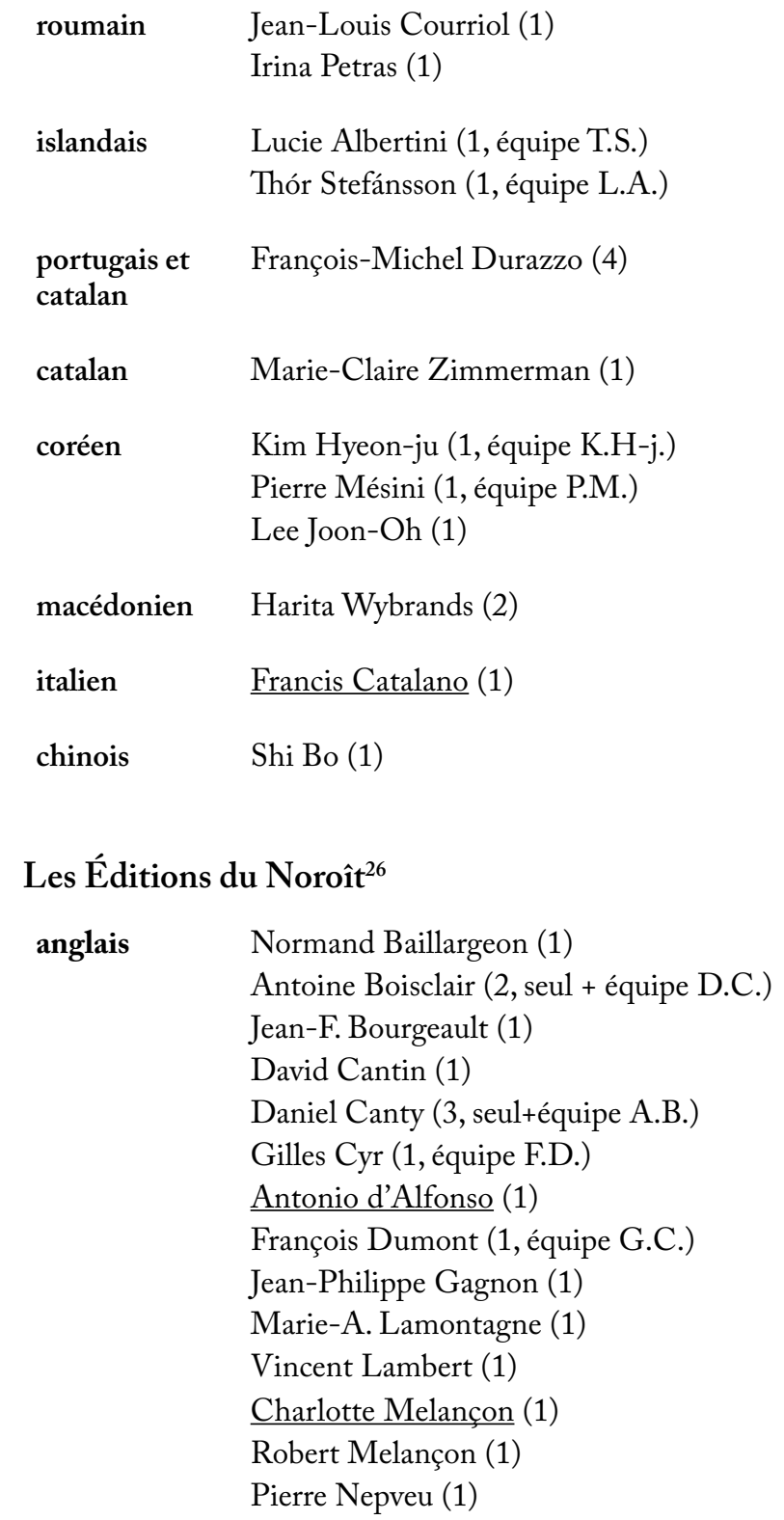

\section{Les Éditions du Noroît ${ }^{26}$}

26. Les traducteurs dont le nom est souligné sont ceux qui figuraient dans la liste des membres actifs de l'ATTLC au 20 octobre 2015. De cette liste, seul Louis Jolicoeur figurait dans le répertoire au 27 mai 2017. 


$\begin{array}{ll}\text { catalan } & \text { Denise Desautels (1) } \\ \text { espagnol } & \text { Nelly Roffé (3) } \\ & \begin{array}{l}\text { François-Michel Durazzo (2) } \\ \text { Louis Jolicoeur (1) }\end{array} \\ & \text { Patrick Lavaud (1) } \\ \text { italien } & \text { Francis Catalano (2) } \\ \text { coréen } & \text { Han Daekyun (1) }\end{array}$

\section{Éditions L'Oie de Cravan}

$\begin{array}{ll}\text { anglais } & \text { Alice Becker-Ho (1) } \\ & \text { Marie Frankland (1) } \\ & \text { Benoît Chaput (1) } \\ \text { grec } & \text { Michel Volkovitch (1) }\end{array}$

\section{Éditions d'Art Le Sabord}

anglais Guy Jean (2) 